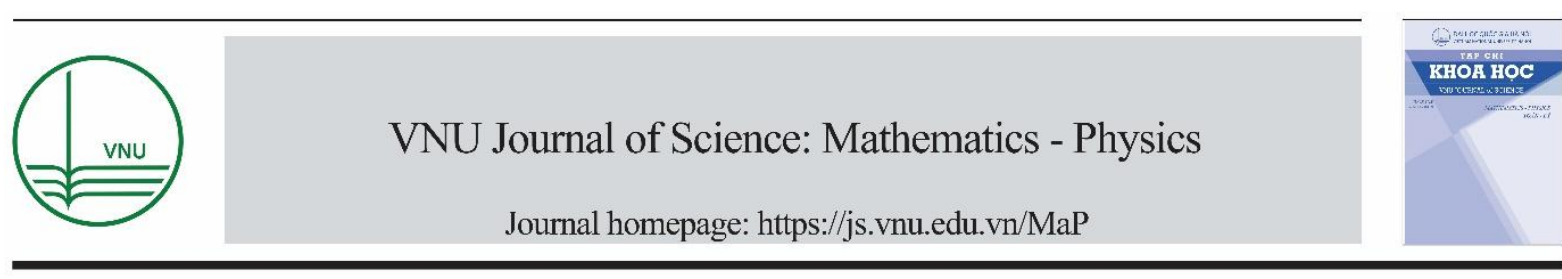

Original Article

\title{
High-pressure Melting Curves of $\alpha$ and $\gamma$ Phases of Iron
}

\author{
Nguyen Thi Hong ${ }^{1,2, *}$, Ho Khac Hieu ${ }^{3}$ \\ ${ }^{1}$ Hong Duc University, 307 Le Lai, Dong Son, Thanh Hoa, Vietnam \\ ${ }^{2}$ VNU University of Science, 334 Nguyen Trai, Hanoi, Vietnam \\ ${ }^{3}$ Duy Tan University, 254 Nguyen Van Linh, Thanh Khe, Da Nang, Vietnam
}

Received 22 September 2019

Revised 11 October 2019; Accepted 15 October2019

\begin{abstract}
In this study, statistical moment method (SMM) was applied in combination with Lindemann melting criterion to investigate pressure effects on melting temperature of iron. Melting curves of $\alpha$ phase with body-centered cubicstructure and $\gamma$ phase with face-centered cubicstructure of iron were derived up to pressure $13 \mathrm{GPa}$ and $90 \mathrm{GPa}$, respectively. The study results show that melting curves of these two phases of iron increased functions of pressure, and the higher the pressure was the lower the slopes of melting curves were. The results were compared with the available experimental data to verify the developed theory. The efficiency of the SMM on the investigation of melting temperatures of $\alpha$ and $\gamma$ phases of iron suggests that the present SMM scheme can be developed extensively to determine melting temperatures of other phases of iron as well as other materials.
\end{abstract}

Keywords: Melting, high pressure, iron, Lindemann criterion, Statistical moment method.

\section{Introduction}

Earth's solid inner core is mainly composed of iron [1]. Iron has four main polymorphs including $\alpha$ phase with body-centered cubic (BCC) structure, $\gamma$ phase with face-centered cubic (FCC) structure, $\varepsilon$ phase with hexagonal close-packed (HCP) structure, and $\delta$ phase with BCC structure at high temperature [2-4]. Moreover, Andrault et al. experimentally showed the persistence of the fifth phase $\beta$ phase of iron at high temperature and pressure [5]. Melting curves of iron in different structural

\footnotetext{
${ }^{*}$ Corresponding author.

Email address: hongnguyenhdu86@gmail.com
}

https//doi.org/ 10.25073/2588-1124/vnumap.4382 
phases at high pressure provide essential information to investigate complex seismic structures inside the Earth's core [6-7]. The investigation of melting of iron under high pressure has been performed by different theoretical methods (ab initio calculations [8], molecular dynamic simulations [9], empirical approach [10-12]) and experimental measurements (laser-heated diamond-anvil cell [13-15], shock compression [16]). However, up to now, the results obtained from different methods are still not unified.

In this work, by developing the statistical moment method (SMM) in statistical mechanics, we investigate the pressure dependence of melting temperatures of $\alpha$ and $\gamma$ phases of iron. The slopes of melting curves have also been derived. Our numerical calculations are compared with those of previous theoretical and empirical works to verify the research approach.

\section{Theoretical approach}

The melting temperature of iron, in present work, is derived based on the Lindemann melting criterion which was proposed that [17-19]: "Melting of a material is going to occur when the so-called Lindemann ratio $\zeta(P, T)=\frac{\sqrt{\left\langle u^{2}\right\rangle}}{a(P, T)}$ reaches a threshold value", where $\left\langle u^{2}\right\rangle$ is the atomic mean-square displacement (MSD) and $a(P, T)$ is the nearest-neighbor distance (NND) between two intermediate atoms.

In order to derive the melting point of iron at different pressures, we propose an assumption that the Lindemann ratio remains constant for all range of studied pressure [20], i.e.:

$$
\zeta(P, T)=\frac{\sqrt{\left\langle u^{2}\right\rangle(P, T)}}{a(P, T)}=\zeta(0, T)=\frac{\sqrt{\left\langle u^{2}\right\rangle(0, T)}}{a_{0}(0, T)},
$$

where $\zeta(0, T)=\frac{\sqrt{\left\langle u^{2}\right\rangle(0, T)}}{a_{0}(0, T)}$ is the Lindemann ratio at ambient pressure and at melting point temperature of the material. This assumption can be seen as an expansion of Lindemann criterion. This comes from a truth that when pressure increases, both MSD and NND quantities will be reduced due to the limitation of atomic vibrations. Below we present a method to determine the NND $a(P, T)$ and $\operatorname{MSD}\left\langle u^{2}\right\rangle$ of atoms.

\subsection{Nearest-neighbor distance}

In SMM approach, the NND $a(P, T)$ was derived as [21]

$$
a(P, T)=a_{0}(P, 0)+y_{0}(P, T),
$$

where $a_{0}(P, 0)$ is NND at $0 \mathrm{~K}, y_{0}(P, T) \approx \sqrt{\frac{2 \gamma \theta^{2}}{3 k^{3}}}$ is the thermally induced lattice expansion at pressure $P$ and temperature $T, \theta=k_{B} T$ with $k_{B}$ is the Boltzmann constant, $k$ and $\gamma$ are the harmonic and anharmonic parameters, respectively. 
The parameters $A, k$, and $\gamma$ were defined as follows [21]

$$
\begin{gathered}
A=a_{1}+\frac{\gamma^{2} \theta^{2}}{k^{4}} a_{2}+\frac{\gamma^{3} \theta^{3}}{k^{6}} a_{3}+\frac{\gamma^{4} \theta^{4}}{k^{8}} a_{4}+\frac{\gamma^{5} \theta^{5}}{k^{10}} a_{5}+\frac{\gamma^{6} \theta^{6}}{k^{12}} a_{6}, \\
k=\frac{1}{2} \sum_{i}\left(\frac{\partial^{2} \varphi_{i 0}}{\partial u_{i \beta}^{2}}\right)_{e q}=m_{0} \omega^{2}, \\
\gamma=\frac{1}{12} \sum_{i}\left[\left(\frac{\partial^{4} \varphi_{i 0}}{\partial u_{i x}^{4}}\right)_{e q}+6 \sum_{i}\left(\frac{\partial^{4} \varphi_{i o}}{\partial u_{i x}^{2} \partial u_{i y}^{2}}\right)_{e q}\right],
\end{gathered}
$$

where $a_{1}, a_{2}, a_{3}, a_{4}, a_{5}, a_{6}$ were defined as in Ref. [21], $\varphi_{i o}$ is the interaction potential between the $i^{\text {th }}$ and the $0^{\text {th }}$ particles, $m_{0}$ is the atomic mass.

The NND $a_{0}(P, 0)$ can be calculated from the SMM equation-of-state (EOS) as [21]

$$
P v=-a\left[\frac{1}{6} \frac{\partial U_{0}}{\partial a}+\theta X \frac{1}{2 k} \frac{\partial k}{\partial a}\right],
$$

where $v$ is the atomic volume, $U_{0}$ is the total potential energy of system, and $X=\left(\frac{\hbar \omega}{2 \theta}\right) \operatorname{coth}\left(\frac{\hbar \omega}{2 \theta}\right)$.

At ambient temperature, the Eq. (5) is reduced to a simple form

$$
P v=-a\left[\frac{1}{6} \frac{\partial U_{0}}{\partial a}+\frac{\hbar \omega_{0}}{4 k} \frac{\partial k}{\partial a}\right] .
$$

In order to perform numerical calculations, we assume the interaction potential between two intermediate atoms could be described by the Lennard-Jones potential as

$$
\varphi(r)=\frac{D}{(n-m)}\left[m\left(\frac{r_{0}}{a}\right)^{n}-n\left(\frac{r_{0}}{a}\right)^{m}\right],
$$

where $D$ describes the dissociation energy, $r_{0}$ is the equilibrium value of $a$, and $n, m$ are determined by fitting experimental data.

Using the coordination sphere method, we derive the total potential energy of system as [22]

$$
U_{0}=\frac{N . D}{2(n-m)}\left[m A_{n}\left(\frac{r_{0}}{a}\right)^{n}-n A_{m}\left(\frac{r_{0}}{a}\right)^{m}\right],
$$

where $N$ is the total number of particles in the crystal.

Substituting $\varphi(r)$ of the Lennard-Jones potential into Eqs. (3) and (4), the parameters $k$ and $\gamma$ are then derived [21] 


$$
\begin{gathered}
k=\frac{D n m}{2 a^{2}(n-m)}\left\{\left[(n+2) A_{n+4}^{a_{\mathrm{ix}}^{2}}-A_{n+2}\right]\left(\frac{r_{0}}{a}\right)^{n}-\right. \\
\left.-\left[(m+2) A_{m+4}^{a_{\mathrm{ix}}^{2}}-A_{m+2}\right]\left(\frac{r_{0}}{a}\right)^{m}\right\}, \\
\gamma=\frac{D m n}{12 a^{4}(n-m)} \times\left\{\left[(n+2)(n+4)(n+6)\left(A_{n+8}^{a_{\mathrm{ix}}^{4}}+6 A_{n+8}^{a_{\mathrm{ix}}^{2} a_{i y}^{2}}\right)-18(n+2)(n+4) A_{n+6}^{a_{\mathrm{ix}}^{2}}\right.\right. \\
\left.+9(n+2) A_{n+4}\right]\left(\frac{r_{0}}{a}\right)^{n}-\left[(m+2)(m+4)(m+6)\left(A_{m+8}^{a_{\mathrm{ix}}^{4}}+6 A_{m+8}^{a_{\mathrm{ix}}^{2} a_{i y}^{2}}\right)-\right. \\
\left.\left.-18(m+2)(m+4) A_{m+6}^{a_{\mathrm{ix}}^{2}}+9(m+2) A_{m+4}\right]\left(\frac{r_{0}}{a}\right)^{m}\right\},
\end{gathered}
$$

where $A_{n}, A_{m}, A_{m}^{a_{i x}^{2}}, A_{n}^{a_{i x}^{2}}, \ldots$ are the structural sums of the given crystal [21,22].

The EOS of crystal at zero temperature is obtained as [21]

$$
\begin{aligned}
P v=-\frac{D n m}{6(n-m)}\left[-A_{n}\left(\frac{r_{0}}{a}\right)^{n}+A_{m}\left(\frac{r_{0}}{a}\right)^{m}\right]-\frac{\hbar}{4 a^{2} \sqrt{M}} \sqrt{\frac{D n m}{2(n-m)}} \times \\
\times \frac{(n+2)\left[(n+2) A_{n+4}^{a_{\mathrm{i}}^{2}}-A_{n+2}\right]\left(\frac{r_{0}}{a}\right)^{n}-(m+2)\left[(m+2) A_{m+4}^{a_{\mathrm{i}}^{2}}-A_{m+2}\right]\left(\frac{r_{0}}{a}\right)^{m}}{\sqrt{\left[(n+2) A_{n+4}^{a_{\mathrm{i}}^{2}}-A_{n+2}\right]\left(\frac{r_{0}}{a}\right)^{n}-\left[(m+2) A_{m+4}^{a_{\mathrm{ix}}^{2}}-A_{m+2}\right]\left(\frac{r_{0}}{a}\right)^{m}}} .
\end{aligned}
$$

This equation can be re-written in a simpler form

$$
c_{1} y^{n+3}-c_{2} y^{m+3}+\frac{c_{3} y^{n+4}-c_{4} y^{m+4}}{\sqrt{c_{5} y^{n}-c_{6} y^{m}}}-P v=0,
$$

where

$$
\begin{aligned}
& y=\frac{r_{0}}{a} ; \quad c_{1}=A_{n} \frac{D n m}{6(n-m)} ; \quad c_{2}=A_{m} \frac{D n m}{6(n-m)} ; \\
& c_{3}=\frac{1}{a} \frac{\hbar}{4 \sqrt{M}} \sqrt{\frac{D n m}{2(n-m)}}(n+2)\left[(n+2) A_{n+4}^{a_{\mathrm{i}}^{2}}-A_{n+2}\right] ; \\
& c_{4}=\frac{1}{a} \frac{\hbar}{4 \sqrt{M}} \sqrt{\frac{D n m}{2(n-m)}}(m+2)\left[(n+2) A_{m+4}^{a_{\mathrm{i}}^{2}}-A_{m+2}\right] ; \\
& c_{5}=(n+2) A_{n+4}^{a_{\mathrm{ix}}^{2}}-A_{n+2} ; \quad c_{6}=(m+2) A_{m+4}^{a_{\mathrm{ix}}^{2}}-A_{m+2} ;
\end{aligned}
$$

$v=\frac{4}{3 \sqrt{3}} r_{0}^{3}$ (for BCC structure) and $v=\frac{\sqrt{2}}{2} r_{0}^{3}$ (for FCC structure). 
By numerically solving Eq. (12), we can determine the value of NND $a_{0}(P, 0)$.

\subsection{Atomic mean-square displacement}

The MSD function can be derived from the second order moment of SMM method as [23]

$$
\left\langle u^{2}\right\rangle=\langle u\rangle^{2}+\theta A_{1}+\frac{\theta}{k}(X-1)
$$

where

$$
\begin{aligned}
& \langle u\rangle \approx y_{0}, \\
& A_{1}=\frac{1}{k}\left[1+\frac{2 \gamma^{2} \theta^{2}}{k^{4}}\left(1+\frac{X}{2}\right)(X+1)\right] .
\end{aligned}
$$

\section{Results and discussion}

In this section, numerical calculations will be performed to evaluate the pressure-dependent melting temperatures of $\alpha$ and $\gamma$ phases of iron. The Lennard-Jones potential parameters of iron metal are $m=$ 3.54, $n=6.45, r_{0}=2.48 \AA, D=12576.7 k_{B}$ [23]. With the assumption that the interatomic potential does not depend on the structure of iron, the above potential could be used for numerical calculations of these two phases of iron.

In order to determine the melting curves of $\alpha$ (in the pressure range $0-13 \mathrm{GPa}$ ) and $\gamma$ (in the pressure range 13-90 GPa) phases of iron, we calculate firstly the Lindemann ratio at zero pressure and at melting point $\left(T_{m 0}=1811 \mathrm{~K}\right)$. The Lindemann ratios of two phases are obtained, respectively, as 0.0589046 and 0.0525603 for $\alpha-\mathrm{Fe}$ and $\gamma$-Fe. From these results and from our assumption (the pressure independence of Lindemann criterion) we derive the melting temperatures of iron at different pressures. Our melting curves of $\alpha$ and $\gamma$ phases of iron are shown in Fig. 1.

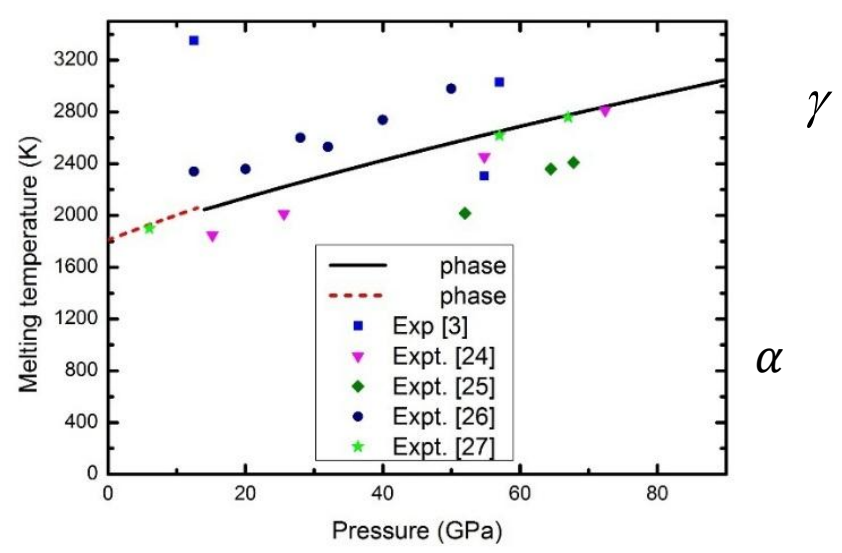

Fig. 1. Melting curves of $\alpha$ and $\gamma$ phases of iron. Our results (solid lines) are compared with those of measurements performed by Anderson [3], Ahrens et al. [24], Komabayashi et al. [25], Jackson et al. [26], and Sinmyo et al. [27]. 
As observed from Fig. 1, the melting curves of $\alpha$ and $\gamma$ phases of iron are increasing functions of pressure. Our calculations of melting temperatures of iron are reasonably consistent with most of those of previous works but they overestimate the measurements performed by Komabayashi et al. [25]. The maximum difference between our predictions and previous works is about $11.68 \%$ at $52 \mathrm{GPa}$. Especially, it should be noted that our theoretical melting curves are in good agreement with the most recently published data measured by Sinmyo et al. [27].

We make a futher step by considering the slopes of melting curves of $\alpha$ and $\gamma$ phases of iron. The slopes of these two melting curves are presented in Fig. 2. As it can be seen from this figure that the higher the pressure is the lower the slopes of melting are. Interestingly, at the pressure of structural phase transition, we can observe the apparent break between two melting curves. The difference between the slopes of two melting curves at pressure $13 \mathrm{GPa}$ is about $2.2 \mathrm{~K} / \mathrm{GPa}$. This break behavior can be applied for the investigation of the structural phase transition of materials under pressure.

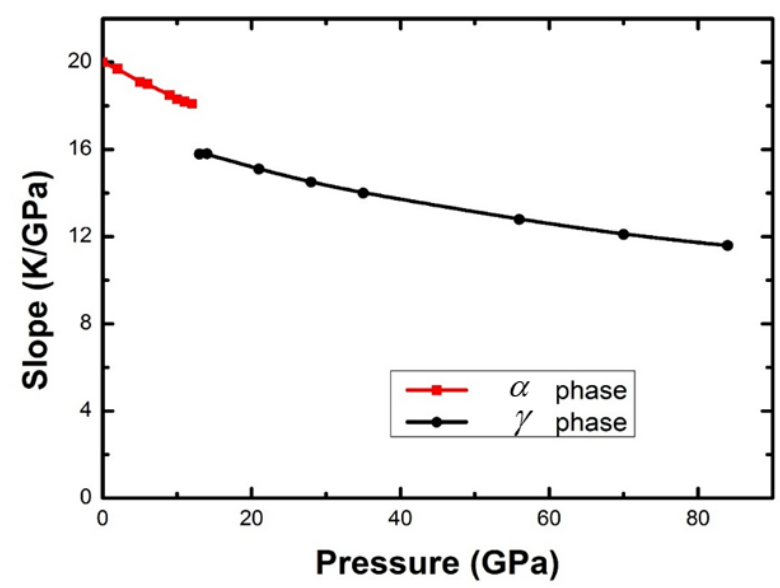

Fig. 2. The slopes of the melting curves of $\alpha$ and $\gamma$ phases of iron.

\section{Conclusions}

In this work, we have studied the melting curves of $\alpha$ and $\gamma$ phases of iron by using the combination of SMM with modified Lindemann criterion of melting. The slopes of melting curves of these two phases of iron have also been derived. Our numerical calculations show that the SMM melting temperatures of these two phases of iron are in reasonable agreement with the available experimental data. This confirms that our approach has a potential to study pressure effects on melting curves of other phases of iron as well as other materials.

\section{Acknowledgments}

This research is funded by the Vietnam National Foundation for Science and Technology Development (NAFOSTED) under grant number 103.01-2017.343. 


\section{References}

[1] Y. Mori, H. Ozawa, K. Hirose, R. Sinmyo, S. Tateno, G. Morard, Y. Ohishi, Melting experiments on Fe-Fe3S system to $254 \mathrm{Gpa}$, Earth Planet. Sci. Lett. 464 (2017) 135-141.

[2] C. S. Yoo, J. Akella, A. J. Campbell, H. K. Mao, R. J. Hemley, Phase Diagram of lron by in Situ X-ray Diffraction: Implications for Earth's Core, Science 270 (1995) 1473-1475.

[3] O. L. Anderson, Iron: Beta Phase Frays, Science 278 (1997) 821-822.

[4] K. Ohta, Y. Kuwayama, K. Shimizu, T. Yagi, K. Hirose, Y. Ohishi, Measurements of electrical and thermal conductivity of iron under Earth's core conditions, AGU abstract MR21B-06, AGU Fall Meeting, San Francisco, (2014) Dec 15-19.

[5] D. Andrault, G. Fiquet, M. Kunz, F. Visocekas, D. Hausermann, The Orthorhombic Structure of iron: An in Situ Study at High-Temperature and High-pressure, Science 278 (1997) 831.

[6] M. Mattesini, A. B. Belonoshko, E. Buforn, M. Ramírez, S. I. Simak, A. Udías, H. K. Mao, and R. Ahuja, Hemispherical anisotropic patterns of the Earth's inner core, Proc. Natl. Acad. Sci. U.S.A. 107 (2010) 9507-9512.

[7] M. Monnereau, M. Calvet, L. Margerin, A. Souriau, Lopsided Growth of Earth's Inner Core, Science 328 (2010) 1014.

[8] A. B. Belonoshko, R. Ahuja, B. Johansson, Stability of the body-centred-cubic phase of iron in the Earth's inner core, Nature 424 (2003) 1032.

[9] Y. Wu, L. Wang, Y. Huang, D. Wang, Melting of copper under high pressures by molecular dynamics simulation, Chem. Phys. Lett. 515 (2011) 217-220.

[10] D. Zhang, J.M. Jackson, J. Zhao, W. Sturhahn, E.E. Alp, M.Y. Hu, T.S. Toellner, C.A. Murphy, V.B. Prakapenka, Temperature of Earth's core constrained from melting of $\mathrm{Fe}$ and $\mathrm{Fe}_{0.9} \mathrm{Ni}_{0.1}$ at high pressures, Earth Planet. Sci. Lett. 447 (2016) 72-83.

[11] H.K. Hieu, Melting of solids under high pressure, Vacuum 109 (2014) 184-186.

[12] H.K. Hieu, T.T. Hai, N.T. Hong, N.D. Sang, N.V. Tuyen, Pressure dependence of melting temperature and shear modulus of hcp-iron, High Pressure Res. 37 (2017) 267-277.

[13] S. Anzellini, A. Dewaele, M. Mezouar, P. Loubeyre, G. Morard, Diffraction Melting of Iron at Earth's Inner Core Boundary Based on Fast X-ray, Science 340 (2013) 464-466.

[14] Q. Williams, R. Jeanloz, J. Bass, B. Svendsen, T.J. Ahrens, The Melting Curve of Iron to 250 Gigapascals: A Constraint on the Temperature at Earth's Center, Science 236 (1987) 181-182.

[15] C.S. Yoo, N.C. Holmes, M. Ross, D.J. Webb, C. Pike, Shock Temperatures and Melting of Iron at Earth Core Conditions, Phys. Rev. Lett. 70 (1993) 3931-3934.

[16] N.H. Jeffrey, H.C. Neil, Melting of iron at the physical conditions of the Earth's core, Nature 427 (2004) $339-342$.

[17] H.K. Hieu, Volume and pressure-dependent thermodynamic properties of sodium, Vacuum. 120 (2015) $13-16$.

[18] H.K. Hieu and N. N. Ha, High pressure melting curves of silver, gold and copper, AIP Adv. 3 (2013) 112125.

[19] F. Lindemann, The calculation of molecular vibration frequencies, Phys. Z. 11 (1910) 609-612.

[20] H.K. Hieu, Systematic prediction of high-pressure melting curves of transition metals, J. Appl. Phys. 116 (2014) 163505-1 - 16305-6.

[21] V.V. Hung, Statistical moment method in studying thermodynamic and elastic property of crystal, HNUE Publishing House, Hanoi, 2009.

[22] V.V. Hung, N.T. Hoa, Equation of state and thermodynamic properties of BCC metals, ASEAN J. Sci. Tech. Dev. 23 (2006) 27-42.

[23] M. Magomedov, The Calculation of the Parameters of the Mie-Lennard-Jones Potential, High Temp. 44 (2006) 513-529.

[24] T.J. Ahrens, K.G. Holland, G.Q. Chen, Phase diagram of iron, revised-core temperatures, Geophys. Res. Lett. 29 (2002) 54-1 - 54-4.

[25] T. Komabayashi, Y.W. Fei, Internally consistent thermodynamic database for iron to the Earth's core conditions, J. Geophys. Res. Solid Earth 115 (2010) B03202.

[26] J.M. Jackson, W. Sturhahn, M. Lerche, J. Zhao, T.S. Toellner, E. E. Alp, S.V. Sinogeikin, J.D. Bass, C.A. Murphy, J.K. Wicks, Melting of compressed iron by monitoring atomic dynamics, Earth Planet. Sci. Lett. 362 (2013) 143150.

[27] R.Sinmyo, K. Hirose, Y. Ohishi, Melting curve of iron to $290 \mathrm{GPa}$ determined in a resistance-heated diamondanvil cell, Earth Planet. Sci. Lett. 510 (2019) 45-52. 